\title{
Discount Rate Water Absorption Plant Fibers / Treatment of Physical Rice Straw by Dumping in Soil Solutions
}

\author{
Department of Earth Sciences and Mineral Resource \\ Geomaterial Laboratory, University Félix Houphouët-Boigny \\ Abidjan, Côte d'Ivoire
}

SERIFOU Mamery Adama, KOUASSI Bleh Raoul, JOLISSAINT Obre Sery Paul and EMERUWA Edjikémé

\begin{abstract}
The rice straw (agricultural waste) was included in the construction of homes in Africa. This application is still useful since it improves some physical properties of materials. However, the great absorption of water of such fibers constitutes an obstacle to the hydration of cement. To remedy this we offer a fiber processing technique. This technique using soil reduces from 15 to $33 \%$ absorption rate of rice straw. The processing performance depends on the soil used.
\end{abstract}

Key Words: Natural fibers, water absorption, processing, materials, agricultural waste.

\section{INTRODUCTION}

The use of agricultural waste in construction is one of the traditional ways in Africa. This is the case of rice straw. They are mixed with black mud to increase its strength and control shrinkage. They serve as reinforcement for the rendering. This practice dates back to the Neolithic [1]. Thus, cob considered the first composite material of history included a plastic matrix (ground) and reinforcements (plant fibers). Environmental issues and sustainable development are at the center of much debate. The management of agricultural waste is also affected by these issues. Their valuation in construction materials through the composite formulation is an effective solution.

Cote d'Ivoire is an agricultural country and rice cultivation is much practiced in the west and south of the country. This activity produces waste including vegetable fibers. Used as reinforcing agents in composite mineral matrix, rice straws is used to reduce the density while improving thermal and acoustic performance of the materials [2].

But one of the disadvantages of using plant fibers in construction materials is the high water absorption rate [3]. Water for hydrated cement could be absorbed by the fibers thus influencing the cement and composite properties. This has led us to initiate a work whose theme is "reduced water absorption rate of plant fiber / physical treatment immersions by rice straw in soil solution". The absorption of the fibers influences the mechanical properties of materials. However, to overcome this drawback various methods of fiber processing exist [4]. These methods as well as physical, chemical and thermal each have advantages and disadvantages. We propose a new method of treatment. For a good presentation, the document will comprise two parts: First we will present materials and methods; then the results, interpretation of our experiments. It ends with a conclusion.

\section{MATERIALS AND METHODS}

\subsection{Raw Material}

\subsubsection{Rice Straw}

Rice is an annual plant glabrous thatched (stem) derived or spread of variable height, ranging from less than one meter up to five meters for floating rice. It's a plant susceptible tillering, forming a drill bouquet, fibrous root [5]. Like other natural fiber, rice fiber is a stack of composite plies reinforced with cellulose fibrils [6]. On the surface of the fibers, exists stomata. Those aremicroscopic openings in the skin to sweat and ensuring the gas exchange between the plant and atmosphere. They cause additional water absorption and promote the absorption and movement of mineral elements [7]. 
The rice straw with which we worked was collected in a plantation in the town of Agboville (south of Côte d'Ivoire). They will pose storage problems in the rice basin since they represent a high level of waste.

\subsubsection{Soil}

We used three types of soil. There is clay, laterite and sand.

- The clay was taken in Dabou (south of Côte d'Ivoire). It is derived from the alteration of a granitoid [8].

- Laterite from a deposit located in the town of Songon, Abidjan in the locality. This laterite is exploited for the construction of mud blocks (BTC) and for the formation of base courses of roads.

- Sand comes from a quarry in the municipality of Port-Bouet (south of Abidjan). It is taken from the lagoon with a dredge. This sand is used mostly in construction.

\subsection{METHODOLOGY}

\subsubsection{Treating Fibers}

The fiber processing was done with soil solutions. These solutions were obtained by mixing the soil with water faucet for a concentration of $100 \mathrm{~g} / \mathrm{l}$. To this solution, is added the rice fiber according to the weight ratio $\mathrm{P} / \mathrm{S}=0.03$ (with $\mathrm{P}$ denoting the straws and S soil). The straws stay in this solution for 24 hours in a covered container. Thereafter, the fibers are dried to a constant mass.

\subsubsection{Determining the Absorption Rate of the Fibers}

The water absorption coefficient of the straw was determined by the method described by [9] in his thesis. The protocol used is as follows:

- $\quad$ Take a dried fiber mass (m1), and soak it in water for 24 hours;

- $\quad$ placing the fibers in a centrifuge and actuate for 30 seconds.

- Weigh the dewatered fibers and note the new mass (m2) of the fibers;

- $\quad$ the value of the water absorption is obtained using the formula (1):

$$
A_{b}=\frac{m_{2}-m_{1}}{m_{1}} \times 100
$$

Initially the value was obtained for untreated fibers; then it was the case of fibers treated with various floor solutions. In the latter case, there is the rate obtained Ab1. The rate of reduction in absorption is then obtained by the following formula:

$$
R_{d}=\frac{A b_{1}-A b}{A b} \times 100
$$

This parameter is calculated for all treatments and results are used to compare the effectiveness of different treatments on reducing water absorption fiber.

\section{RESULTS AND DISCUSSION}

\subsection{Absorption Rate}

The masses of dry fibers and those of fibers soaked for twenty-four hours were obtained. The Figure 1 shows the results as a strip chart.

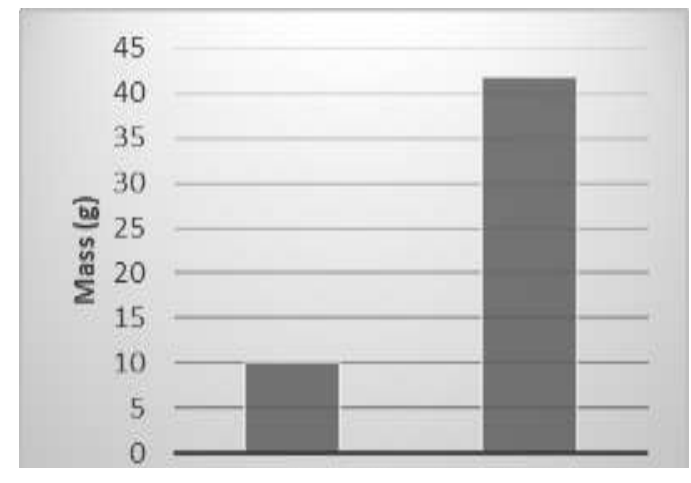

Before Immersion After Immersion

Figure1. Rice straw untreated masses before and after immersion 


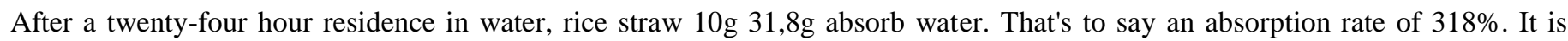
noted that rice straw absorbs more than three times its weight in water. This great absorption is observed in other plants such as aggregate boon that can absorb up to 4 times its weight in water $(400 \%)$ before reaching full saturation [2]; also the date palm fibers which absorb three times their dry weight [10]. Other fibers have lower absorption rates. This is the case for example of flax fibers with an absorption rate of $130 \%$. This high capacity of water absorption of the fibers could be due to a large amount of stomata on the surface of these fibers. Indeed, the stomata are the interface for communication with the external environment. These exchanges take place at the plant physiological activities such as breathing, sweating, etc.

\subsection{Reduction Rate of Absorption}

The rate of reduction of fiber water absorption was obtained for each treatment and the results are presented in Figure 2.

Sand $\quad$ Laterite $\quad$ Clay

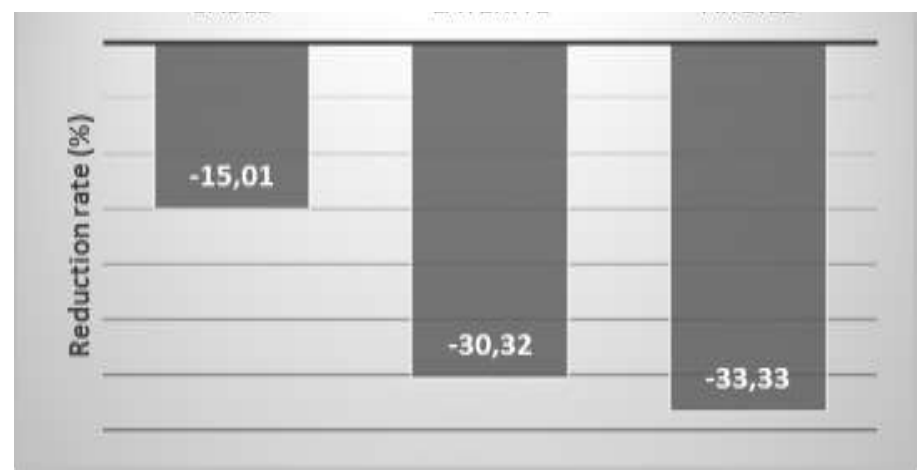

Figure 2: the absorption of water reduction rate by treatment

Treating the fibers by the physical channel soaking in soil solutions effectively reduces the rate of absorption of plant fibers. The best result was obtained with a clay solution.

Other authors have used clay to reduce the water absorption rate. So, in macro-nano-composites low density polyethylenes, a significant reduction was observed in the water absorption of the composite in the presence of clay. The incorporation of 5\% clay composite in a divided by three (3) water absorption [6]. These results illustrate the effect of the clay on the water absorption of the material containing plant fibers. This could be explained by the fact that sticky clay adheres to the membrane fibers and arrives at block stomata (pores). They constitute the fiber water absorption channel [7]. Thus, the clay particles reduce the voids available for storing water [11].

It is also observed that the effectiveness of the treatment depends on the fineness of soil particles in solution. Clay treatment (thinner) acts more efficiently as laterite and has finally the one with the sand (coarser). The action of the particles is to reduce the porosity of the fibers. It is the pores that are the basis of strong water absorption rate [10]. Other authors have also thought to reduce the rate of fiber's water absorption by reducing their porosity. For example Sil wood the method uses the micro-silica resulting from the manufacturing of silicon. The wood pores are clogged with micro-silica. This slows the absorption effect of the wood. The implementation of this process is nevertheless limited by the high cost of micro-silica [12].

The use of water for soaking could also be the cause of this improvement [13] also observed reductions in water absorption rate with the soaking in sea water and tap water. However, the fibers treated with tap water have a lower absorption rate.

\section{CONCLUSION}

The use of vegetable fibers in building materials is a very interesting alternative for the management of agricultural waste. They improve certain physical properties of composites. But the high water absorption of the fibers is a problem particularly for cement hydration. Rice straw absorbs more than three times their weight in water. To provide a solution to the experiment was conducted a soaking of these fibers in three soil solutions (sand, laterite, clay). This treatment effectively reduces the rate of water absorption of the fibers according to the soil solution used. The best result was obtained with the clay with a reduction rate of about $33 \%$. It would be interesting to study the impact of these treatments on the interface fiber/cement.

\section{REFERENCES}

1. D. Sedan Study of physico-chemical interactions with the hemp / cement fiber interfaces. Influence on the mechanical properties of the composite, PhD Thesis, University of Limoges, France, 2007, 137p. 
2. C. Morgan. Formulation and study of the mechanical properties of lightweight insulating concrete agro-based rice balls and boon for green building, PhD Thesis, University of Montpellier, France, 2016, 226p.

3. V. Cerezo. Mechanical, thermal and acoustic properties of a base material of vegetable particles: experimental approach and theoretical modeling. PhD thesis, National School of Public Works of the State, France, 2005, 243p.

4. R. Osmani and Mr. H. The effect of surface treatment of composite fibers on the mechanical properties Alfa Polyester fibers. In the 20th French Congress of Mechanics, Besancon, 29 August to 2 September 2011, 2011, pp. 1-6.

5. R. R. Nambinina, J. R. Haritiana, R Heriniaina, F. Randrianarivelo, T.R. Randriamalala. Strengthening Concrete Structure of Earth Tablet (BTC) by inserting the fiber, journal article, MADA-HARY, ISSN 2410-0315, Vol. 4, 2015, 2015, pp 95-103.

6. R. Harrache. Preparation and characterization matrix composites of polymers and vegetable and laminar nanometric reinforcements. PhD Thesis, University Ferhat Abbas Setif, Algeria, 2015, 132p.

7. N. N. Bachtarzi. The adaptation to water stress in durum wheat (Triticum durum Desf): Screening of physiological and biochemical criteria Master's thesis, University of Mentouri Brothers Constantine, 2015, Algeria, 67p.

8. C. H. Kouakou. Recovery of clays Côte d'Ivoire : study of the stabilization of compressed and stabilized clay blocks cement. PhD Thesis, University Felix Houphouet Boigny, Ivory Coast,2004, 186p.

9. C. Magniont. Contribution to the formulation and characterization of an eco building material made of agricultural resources. PhD Thesis, University of Toulouse, France, 2010, 343p.

10. N. Benmansour. Development and characterization of local natural composite adapted to the thermal insulation in homes. PhD Thesis, University Hadj Lakhdar Batna, 2015, Algeria, 140p

11. D. VVT. Composite natural fiber / biodegradable polymer or not. PhD Thesis, University of Grenoble, France, 2016, $189 \mathrm{p}$.

12. A. Govin. physicochemical aspect of the interaction wood-cement / Modification of cement hydration by wood, PhD, University Jean Monnet in Saint Etienne, France, 2004, 216p.

13. A. Sellami. Development of cementitious composites based on local plant fibers (the diss): Characterization, Sustainability and Application to masonry, PhD, University BADJI Mokhtar-Annaba, Algeria, 2015, 152p. 